TRANSACTIONS OF THE

AMERICAN MATHEMATICAL SOCIETY

Volume 355, Number 8, Pages 3175-3180

S 0002-9947(03)03305-1

Article electronically published on April 17, 2003

\title{
SESHADRI CONSTANTS ON JACOBIAN OF CURVES
}

\author{
JIAN KONG
}

\begin{abstract}
We compute the Seshadri constants on the Jacobian of hyperelliptic curves, as well as of curves with genus three and four. For higher genus curves we conclude that if the Seshadri constants of their Jacobian are less than 2 , then the curves must be hyperelliptic.
\end{abstract}

\section{Introduction and Statement of Results}

Let $X$ be a smooth complex projective variety. Let $L$ be an ample line bundle. Let $p \in X$ be a point. Define the Seshadri constant of $L$ at $p$ to be the real number

$$
\epsilon(L, p):=\inf \left\{\frac{C \cdot L}{\operatorname{mult}_{p} C} \mid p \in C \subset X\right\} .
$$

Here the infimum is taken over all reduced curves $C$ passing through $p$, and mult ${ }_{p} C$ is the multiplicity of $C$ at $p$. Another equivalent definition is

$$
\epsilon(L, p)=\sup \left\{\epsilon \mid f^{*} L-\epsilon E \text { is nef }\right\},
$$

where $f: B l_{p} X \rightarrow X$ is the blow-up of $X$ at $p$ and $E$ is the exceptional divisor.

The Seshadri constant indicates how far the ample divisor is from the boundary of the ample cone near point $p$, and thus measures positivity, or ampleness locally. The study of Seshadri constants has drawn increasing interest during recent years. For properties of Seshadri constants see [1] and [6].

In the case of abelian varieties, it is known that a general element in the moduli space of principally polarized abelian varieties of dimension $g$ has Seshadri constant very close to its maximum upper bound ([4]). On the other hand, there are some special abelian varieties, namely Jacobian, which have relatively small Seshadri constants. We will discuss some cases in this paper.

Let $C$ be a smooth projective algebraic curve over $\mathbf{C}$ with genus $g=g(C) \geq 2$. Denote $\Theta$ to be the theta divisor of $J(C)$, its Jacobian (recall $J(C)=\operatorname{Pic}^{0}(C)$ ). Since abelian varieties are homogeneous spaces, we can define $\epsilon=\epsilon(\Theta, 0)=\epsilon(\Theta, p)$ for any $p \in J(C)$.

It is known that $1<\epsilon \leq \sqrt{g}$, and if $C$ is hyperelliptic, then $\epsilon \leq \frac{2 g}{g+1}([4]$, [5]). In particular, if $g=2$, then $C$ is hyperelliptic and it is known that $\epsilon=\frac{4}{3}([6])$. The problem becomes very interesting even when $g=3$. The point here is to see if the Seshadri constants can be their maximum, i.e., $\sqrt{g}$, thus most of the time irrational, or always less than their maximum - and thus more likely rational. While all the

Received by the editors August 1, 2002 and, in revised form, August 26, 2002.

2000 Mathematics Subject Classification. Primary 14H40; Secondary 14K12.

Key words and phrases. Algebraic geometry, algebraic curves, abelian varieties. 
existing examples suggest the latter, we investigate this problem in detail, mainly by looking at the cases when $\epsilon \leq 2$.

Our main result is the following theorem:

Theorem 1.1. Assume the Picard number of $J(C)$ is one. Then:

(1) If $C$ is hyperelliptic, then $\epsilon=\frac{2 g}{g+1}$.

(2) If $g=3$ and $C$ is not hyperelliptic, then $\epsilon=\frac{12}{7}$.

(3) If $g=4$ and $C$ is not hyperelliptic, then $\epsilon=2$.

(4) If $g \geq 5$ and $C$ is not hyperelliptic, then $\epsilon \geq 2$.

Part (4) of the theorem can be restated as:

Corollary 1.2. If $g \geq 5$ and $\epsilon<2$, then $C$ is hyperelliptic.

Remark 1.3. (1) For the ease of calculation on the Neron-Severi group of the symmetric product $C_{2}$, we need that it is generated by a fiber and the diagonal, i.e., its Picard number is 2 . That is true if $C$ is of general moduli. We need this condition throughout this paper. But this restriction, however, seems to be not essential.

(2) We can also locate all the special curves that give relatively small ratios in cases (1) to (3).

\section{Proof of Theorem: Hyperelliptic Case}

The following observation, while straightforward, points out where we want to find special curves that give the exact value of the Seshadri constants.

Lemma 2.1. If $C^{\prime}$ is an irreducible curve in $J(C)$ such that $\frac{C^{\prime} \cdot \Theta}{\text { mult }_{0} C^{\prime}} \leq 2$, then for any divisor $D$ with $D \equiv k \Theta$ and mult $_{0} D \geq 2 k$, we have $C^{\prime} \subset D$.

If $C$ is hyperelliptic, then the case of $k=1$ in Lemma 2.1 reads $D \equiv \Theta$ and mult $_{0} D \geq 2$, which we denote as $\left(^{*}\right)$. For $d \geq 2$, let $C_{d}$ be the $d$-fold symmetric product of $C$ (the set of effective divisors of degree $d$ on $C$ ).

Proposition 2.2. Let $u: C_{d} \rightarrow J(C)$ be the Abel-Jacobi map. Then

$$
\bigcap_{(*)} D=u\left(C_{2}\right) \text {. }
$$

Proof. Let $L$ be a hyperelliptic line bundle on $C$. Let $p_{0}$ be a ramification point of the $g_{2}^{1}$; so $L=\mathcal{O}_{C}\left(2 p_{0}\right)$. We fix a translation of the Abel-Jacobi map $u: C_{d} \rightarrow J(C)$ by sending $Y \in C_{d}$ to $Y-\operatorname{deg}(Y) \cdot p_{0} \in J(C)$, and for simplicity we ignore the $p_{0}$ part for representation of points in $J(C)$ in our proof. Recall that $\phi: C_{g-3} \rightarrow$ $C_{g-1}, Y \rightarrow Y+L$ maps $C_{g-3}$ birationally and surjectively to $\operatorname{Sing}(\Theta)$ in this case (3]).

For any $Y \in C_{g-3}$, define $D_{Y}=\Theta-Y$. It translates $Y+2 p_{0} \in \operatorname{Sing}(\Theta)$ to $0 \in D_{Y}$. Thus $D_{Y} \equiv \Theta$ and mult $_{0} D \geq 2$. It suffices to show that $\bigcap D_{Y}=u\left(C_{2}\right)$.

It is obvious that $u\left(C_{2}\right) \subset \bigcap D_{Y}$, since for any point $(p, q) \in C_{2}$ we can rewrite it as $(p+q+Y)-Y \in D_{Y}$ for any $Y \in C_{g-3}$.

On the other side, any points in $\bigcap D_{Y}$ can be represented as $D-Y$ for some $D \in C_{g-1}$ and $Y \in C_{g-3}$. Also, since it is in the intersection, for any $F \in C_{g-3}$, there exists $E \in C_{g-1}$ such that $D-Y=E-F$, i.e., $D-Y+F$ is (equivalent to) an effective divisor for any $F$. We claim $D-Y$ itself must be effective, and since it has degree 2 , it is in $u\left(C_{2}\right)$. 
Pick a representation of $D-Y$ such that $Y$ contains no ramification point of $g_{2}^{1}$. First assume that $D$ contains no hyperelliptic pair. If $D-Y$ is not effective, pick $p \in Y$ but $p \notin D$, and let $L=\mathcal{O}_{C}\left(p+p^{\prime}\right)$. Choose $F=Y-p+p^{\prime}$. Then $D-Y+F=D-p+p^{\prime}$. But the linear system $\left|D-p+p^{\prime}\right|$ is empty, since otherwise it must contain a multiple of hyperelliptic pairs and base points, which will lead to $p \in D$.

If $D$ has some hyperelliptic pairs, then cancel as many points in $D-Y$ as possible until either $D-Y$ is effective or $D$ runs out of hyperelliptic pairs and reduce to a similar situation in the first case.

If $C$ is hyperelliptic, and $\operatorname{rk}\left(N S\left(C_{2}\right)\right)=2$, then $N S\left(C_{2}\right)$ is generated by $F$ and $\Delta$, the image of a fiber and the diagonal from the natural map $C \times C \rightarrow C_{2}$. There is a rational curve, call it $\mathbf{P}^{1}$, that consists of hyperelliptic pairs $\{(p, q) \in$ $\left.C_{2} \mid \mathcal{O}_{C}(p+q)=L\right\}$. Also denote $u^{*}(\Theta)$ still as $\Theta$. We list the numerical properties of $N S\left(C_{2}\right)$ below.

Lemma 2.3. With notation as above, we have:

(1) $\Theta=(g+1) F-\frac{1}{2} \Delta$ and $\mathbf{P}^{1}=2 F-\frac{1}{2} \Delta$.

(2) $F^{2}=1, F \cdot \Delta=2, \Delta^{2}=4-4 g$.

The Abel-Jacobi map $u: C_{2} \rightarrow J(C)$ contracts $\mathbf{P}^{1}$ and is isomorphic outside of $\mathbf{P}^{1}$. Now let $C^{\prime \prime}$ be an irreducible curve in $C_{2}$ not contracted by $u$ and let $C^{\prime}=u\left(C^{\prime \prime}\right)$. Then

$$
\frac{C^{\prime \prime} \cdot \Theta}{C^{\prime \prime} \cdot \mathbf{P}^{1}}=\frac{C^{\prime} \cdot \Theta}{\operatorname{mult}_{0} C^{\prime}}
$$

So our theorem in the hyperelliptic case follows from the following proposition.

Proposition 2.4. Among all irreducible curves in $C_{2}$ not contracted by $u, \Delta$ is the only curve with minimum ratio $\frac{\Delta \cdot \Theta}{\Delta \cdot \mathbf{P}^{1}}=\frac{2 g}{g+1}$.

Proof. Since $\Delta \cdot \Theta=4 g$ and $\Delta \cdot \mathbf{P}^{1}=2 g+2$, we have $\frac{\Delta \cdot \Theta}{\Delta \cdot \mathbf{P}^{1}}=\frac{4 g}{2 g+2}=\frac{2 g}{g+1}$.

Let $C_{0}=a F+b \Delta \subset C_{2}$ be an irreducible curve not contracted by $u$. Then $C_{0} \cdot \mathbf{P}^{1}=a+(2 g+2) b \geq 0$ and $C_{0} \cdot \Theta=(a+4 b) g>0$. Then

$$
\frac{\Delta \cdot \Theta}{\Delta \cdot \mathbf{P}^{1}}=\frac{(a+4 b) g}{a+(2 g+2) b}>\frac{2 g}{g+1} \Longleftrightarrow a>0 .
$$

But if $a \leq 0$, then we must have $b>0$ since $C_{0} \cdot \Theta=(a+4 b) g>0$. Now we have $C_{0} \cdot \Delta=2 a+b(4-4 g)<0$. Since both $C_{0}$ and $\Delta$ are irreducible, $C_{0}=\Delta$.

Remark 2.5. A little more detailed calculation shows that $\Delta$ is actually the only curve whose corresponding ratio is less than two.

\section{Proof of the Theorem: Non-hyperelliptic Case}

If $C$ is non-hyperelliptic, then choose the case $k=2$ in Lemma 2.1 which reads $D \equiv 2 \Theta$ and mult $_{0} D \geq 4$. Denote this linear system as $|2 \Theta|_{00}$. So we look at the base locus of $|2 \Theta|_{00}$. Here we need the following result of Welters.

Proposition 3.1 (Welters [7]). $B s\left(|2 \Theta|_{00}\right)=\lambda(C \times C)$. Here $\lambda: C \times C \rightarrow J(C)$, $\lambda(p, q)=p-q$, is the difference map.

Remark 3.2. Welters' theorem is true for all curves with $g=3$ or $g \geq 5$. For $g=4$ the base locus has two more isolated points, which will not affect our proof since we are looking at curves inside the base locus. 
In this case we look at the Neron-Severi group in $C \times C$. It is generated by fibers $F_{1}, F_{2}$ and the diagonal $\Delta$. We list their numerical properties below.

Lemma 3.3. With notation as above, we have:

(1) $\lambda^{*} \Theta=(g-1)\left(F_{1}+F_{2}\right)+\Delta$.

(2) $F_{i}^{2}=0, F_{1} \cdot F_{2}=F_{i} \cdot \Delta=1, \Delta^{2}=2-2 g, i=1,2$.

Since $C$ is non-hyperelliptic, the difference map $\lambda$ contracts the diagonal $\Delta$ to $0 \in J(C)$ and is isomorphic outside $\Delta$. This enables us, as similarly in the hyperelliptic case, to shift the computation from the ratio $\frac{C \cdot \Theta}{\text { multo } C}$ in $J(C)$ to the ratio of the intersection numbers in the Neron-Severi group, which are well understood. Specifically, let $C^{\prime \prime}$ be an irreducible curve in $C \times C$ not contracted by $\lambda$ and let $C^{\prime}=u\left(C^{\prime \prime}\right)$. Then

$$
\frac{C^{\prime \prime} \cdot \lambda^{*} \Theta}{C^{\prime \prime} \cdot \Delta}=\frac{C^{\prime} \cdot \Theta}{\text { mult }_{0} C^{\prime}}
$$

So our theorem in the non-hyperelliptic case follows from the following proposition.

Proposition 3.4. With notation as above:

(1) If $g=3$, the minimum ratio $\frac{C^{\prime \prime} \cdot \lambda^{*} \Theta}{C^{\prime \prime} \cdot \Delta}$ is $\frac{12}{7}$ for curves in $C \times C$, and is achieved by one curve.

(2) If $g=4$, the minimum ratio $\frac{C^{\prime \prime} \cdot \lambda^{*} \Theta}{C^{\prime \prime} \cdot \Delta}$ is 2 for curves in $C \times C$, and is achieved by more than one curve.

(3) If $g \geq 5$, then $\frac{C^{\prime \prime} \cdot \lambda^{*} \Theta}{C^{\prime \prime} \cdot \Delta} \geq 2$ for all curves in $C \times C$ not contracted by $\lambda$.

Proof. (1) $g=3$ : In this case, the canonical system embeds $C$ as a plane quartic. Let $\mathcal{O}_{C}(1)$ be its hyperplane section. Consider the curve

$$
C_{0}=\left\{(p, q) \mid \mathcal{O}_{C}(p+q+2 r)=\mathcal{O}_{C}(1) \text { for some } r \in C\right\} \subset C_{2} .
$$

Write $C_{0}=a F+b \Delta$. Since $C_{0} \cdot \Delta=56$ (twice the number of bitangents) and $C_{0} \cdot F=10$ (degree of the ramification divisor of the dual curve's $g_{3}^{1}$ ), we can solve for $a$ and $b$ and get $C_{0}=16 F-3 \Delta$. $C_{0}$ is irreducible since it is isomorphic to $C$ via $p+q \rightarrow r$. Pulling it back to $C \times C$ we get a curve $C_{0}^{\prime \prime}=16\left(F_{1}+F_{2}\right)+6 \Delta$. Now

$$
\frac{C_{0}^{\prime \prime} \cdot \lambda^{*} \Theta}{C_{0}^{\prime \prime} \cdot \Delta}=\frac{\left[16\left(F_{1}+F_{2}\right)-6 \Delta\right] \cdot\left[2\left(F_{1}+F_{2}\right)+\Delta\right]}{\left[16\left(F_{1}+F_{2}\right)-6 \Delta\right] \cdot \Delta}=\frac{96}{56}=\frac{12}{7} .
$$

To claim that $\frac{12}{7}$ is the minimum ratio, let $C^{\prime \prime}=a F_{1}+b F_{2}+C \Delta$ be any irreducible curve in $C \times C$ not contracted by $\lambda$. If $C^{\prime \prime} \neq C_{0}^{\prime \prime}$, then $C^{\prime \prime} \cdot C_{0}^{\prime \prime}=$ $10(a+b)+56 c \geq 0$. So if $c \geq 0$, then

$$
\frac{C^{\prime \prime} \cdot \lambda^{*} \Theta}{C^{\prime \prime} \cdot \Delta}=\frac{3(a+b)}{a+b-4 c} \geq 3>\frac{12}{7} \text {. }
$$

If $c<0$, then

$$
\frac{C^{\prime \prime} \cdot \lambda^{*} \Theta}{C^{\prime \prime} \cdot \Delta}=\frac{3(a+b)}{a+b-4 c} \geq \frac{3(a+b)}{a+b+\frac{5}{7}(a+b)}=\frac{7}{4}>\frac{12}{7} .
$$

This shows that the only curve that achieves the minimum ratio $\frac{12}{7}$ is $C_{0}^{\prime \prime}$.

(2) $g=4$ : In this case $C$ has two $g_{3}^{1}$ 's. Let $L$ be one $g_{3}^{1}$. Consider the curve $C_{0}=\{(p, q)|| L-p-q \mid>0\} \subset C_{2}$. Since $C_{0} \cdot F=2$ and $C_{0} \cdot \Delta=12$ (degree 
of ramification divisor of $L$ ), we find that $C_{0}=3 F-\frac{1}{2} \Delta$. Lift to $C \times C$ to get $C_{0}^{\prime \prime}=3\left(F_{1}+F_{2}\right)-\Delta$. A similar calculation as above shows that

$$
\frac{C_{0}^{\prime \prime} \cdot \lambda^{*} \Theta}{C_{0}^{\prime \prime} \cdot \Delta}=\frac{24}{12}=2,
$$

and it is the minimum ratio that can be achieved on $C \times C$.

Note that in this case there is another curve (from the other $g_{3}^{1}$ ) that gives the minimum ratio. The reason is that in this case $C_{0}^{2}=0$, while in the case of $g=3$ we have $C_{0}^{2}<0$ (thus unique).

(3) $g \geq 5$ : Assume $C$ has a $g_{d}^{1}(d \geq 3)$, call it $L$. As in (2), consider the curve $C_{0}=\{(p, q)|| L-p-q \mid>0\} \subset C_{2}$. Then $C_{0} \cdot F=d-1$ and $C_{0} \cdot \Delta=2 d+2 g-2$ (degree of ramification divisor of $L$ ). Thus $C_{0}=d F-\frac{1}{2} \Delta$. Lifting to $C \times C$ we get $C_{0}^{\prime \prime}=d\left(F_{1}+F_{2}\right)-\Delta$. Now first we have

$$
\frac{C_{0}^{\prime \prime} \cdot \lambda^{*} \Theta}{C_{0}^{\prime \prime} \cdot \Delta}=\frac{d g}{d+g-1}>2
$$

Secondly, for any irreducible $C^{\prime \prime}=a F_{1}+b F_{2}+c \Delta \subset C \times C$ not contracted by $\lambda$, either $C^{\prime \prime} \cdot C_{0}^{\prime \prime}<0$, or

$$
\frac{C^{\prime \prime} \cdot \lambda^{*} \Theta}{C^{\prime \prime} \cdot \Delta} \geq \frac{d+g-1}{d} \geq 2 \text { if } d \leq g-1 .
$$

Since the Brill-Noether number for $g_{d}^{1}$ is non-negative if $d \geq \frac{g+2}{2}$, both ratios above are at least 2 . If the minimum ratio $\frac{C^{\prime \prime} \cdot \lambda^{*} \Theta}{C^{\prime \prime} \cdot \Delta}<2$, then all the curves $C_{0}$ must be reducible and contain an irreducible component $C_{1}$ whose lift to $C \times C$ gives a small ratio. It is easy to see that $C_{1}^{2}<0$, thus unique in $C_{2}$. This is certainly impossible. (For example, if there are two different $g_{d}^{1}$ for some $d$, then to have a common component for corresponding $C_{0} \subset C_{2}$, one coordinate has to be a base point of $g_{d}^{1}$. Thus it is a linear combination of fibers, and since the component is irreducible, it is a fiber. But for a fiber, the corresponding ratio is $g>2$.)

Note that the minimum ratio exists and can be achieved if $\frac{d g}{d+g-1} \leq \frac{d+g-1}{d}$, which is equivalent to $\frac{d g}{d+g-1} \leq \sqrt{g}$, or $d \leq \sqrt{g}+1$.

\section{Other Related Problems of Seshadri Constants}

For non-hyperelliptic cases when $g \geq 5$, to find the Seshadri constants, the first step is to look at the curves in $C_{2}$. It is related to the problem whether the cone of effective curves of $C_{2}$ is closed. If it is, the curve from the boundary will give a better upper bound of $\epsilon(\Theta)$ which is less than $\sqrt{g}$. In all special cases that we have discussed (hyperelliptic, small genus, curves with $g_{d}^{1}$ for small $d$ ), the cone is closed. For the general case there is some indication that the curve from one boundary (the other one being the diagonal), if closed, will give the ratio $\frac{g q}{p}$ where $(p, q)$ is the primitive solution of Pell's equation $x^{2}-g y^{2}=1$, if $g$ is not a square. The following example gives some indication that it could be true.

Example 4.1. If $C$ is a plane quintic (i.e., genus is 6 ), consider the curve $C_{0}=$ $\left\{(p, q)|| \mathcal{O}_{C}(1)-p-q-2 r \mid>0\right\} \subset C_{2}$. Then $C_{0}$ is irreducible and $C_{0}=50 F-7 \Delta$. Since any curve $C^{\prime} \subset C_{2}$ satisfies $C_{0} \cdot C^{\prime} \geq 0$, a calculation shows that on $C \times C$, $\frac{C^{\prime \prime} \cdot \lambda^{*} \Theta}{C^{\prime \prime} \cdot \Delta} \geq \frac{12}{5}$ holds for all irreducible curves (except $\Delta$ ). Note that the bound $\frac{12}{5}$ is 
what the conjecture gives. Also note that one expects small values for plane curves that are special in the moduli of curves, so general curves of genus 6 must also satisfy that bound.

For $g \geq 5$, if $\epsilon<2$, then it follows that $C$ is hyperelliptic. In all cases, hyperelliptic curves give us the smallest Seshadri constants. From the known result ([2]) of $B s\left(|2 \Theta|_{00}\right)$ in dimension 4, it is easy to see that:

If $A$ is an indecomposable principally polarized abelian variety of dimension 4 and $\epsilon(\Theta)<2$, then $A$ is the Jacobian of a hyperelliptic curve $C$ of genus 4 .

It is very reasonable to ask the same question for any genus and seems like it could be true.

\section{ACKNOWLEDGEMENT}

The author thanks Aaron Bertram for his encouragement and help.

\section{REFERENCES}

1. L. Ein and R. Lazarsfeld, Seshadri constants on smooth surfaces, Journées de Géométrie Algébrique d'Orsay (Orsay, 1992), Astérisque 218 (1993), 177-186. MR 95f:14031

2. E. Izadi, The geometric structure of $A_{4}$, the structure of the Prym map, double solids and $\Gamma_{00}$-divisors, J. Reine Angew. Mathematik 462 (1995), 93-158. MR 96d:14042

3. H. Lange and C. Birkenhake, Complex abelian varieties, Grundlehren der Mathematischen Wissenschaften, Springer-Verlag, Berlin, 1992. MR 94j:14001

4. R. Lazarsfeld, Lengths of periods and Seshadri constants of abelian varieties, Math. Res. Letters 3 (1996), 439-447. MR 98e:14044

5. M. Nakamaye, Seshadri constants on abelian varieties, Amer. J. Math. 118 (1996), 621-635. MR 97k:14005

6. A. Steffens, Remarks on Seshadri constants, Math. Z. 227 (1998), 505-510. MR 99c:14009

7. G. Welters, The surfaces $C-C$ on Jacobi varieties and second order theta functions, Acta Math. 157 (1986), 1-22. MR 87j:14048

Department of Mathematics, Johns Hopkins University, Baltimore, Maryland 21218

E-mail address: jkong@math.jhu.edu 